\title{
Epidemiología de la Insuficiencia Renal Aguda grave. Un estudio prospectivo multicéntrico en la Región Metropolitana
}

\author{
Antonio Vukusich $\mathrm{C}^{1,2,4}$, Felipe Alvear $\mathrm{M}^{\mathrm{a}}$, \\ Pablo Villanueva $A^{a}$, Claudio González $T^{2}$, \\ Francisco Olivari $\mathbf{P}^{3}$, Nelly Alvarado $\mathrm{A}^{3}$, Carlos Zehnder $\mathrm{B}^{4}$. \\ Epidemiology of severe acute renal \\ failure in Metropolitan Santiago
}

Background: There is a paucity of information about the epidemiology of acute renal failure in Chile. Aim: To perform a prospective multicentric survey of severe acute renal failure in Chile. Material and methods: All patients admitted to ten hospitals in Metropolitan Santiago, during a period of six months with severe acute renal failure, were studied. The criteria for severity was the requirement of renal replacement therapy. All patients information was gathered in special forms and the type of renal replacement therapy and evolution was registeres. Results: One hundred fourteen patients were studied (65 males, age range 18 to 87 years). The calculated incidence of acute renal failure was 1.03 cases per 1000 hospital discharges. The onset was nosocomial in 79 subjects (69\%) and community acquired in the rest. Renal failure was oliguric in 64 cases (56\%) and in $60 \%$ of patients it had two or more causative factors. Sepsis, isolated or combined with other causes, was present in 51 of patients. Other causes included ischemia in $47 \%$, surgery in $26 \%$, exogenous toxicity in $25 \%$, endocenous toxicity in $11 \%$, acute glomerular damage in $6 \%$ and obstructive uropathy in $6 \%$. Cardiac surgery was responsible for $47 \%$ of post operative cases of acute renal failure. Intermittent conventional hemodialysis, continuous renal replacement techniques and daily prolonged hemodialysis were used in $66 \%, 29 \%$ and $2 \%$ of patients, respectively. Overall mortality was $45 \%$ and it was higher in oliguric patients. Gender, age, cause or the type of therapy did not influence survival. Nine percent of surviving patients had some degree of kidney dysfunction at discharge. Conclusions: There is still a great space for prevention of severe acute renal failure in Chile, considering the main etiologies found in this study (Rev Méd Chile 2004; 132: 1355-61).

(Key Words: Kidney failure, acute; Oliguria; Renal dialysis)

Recibido el 13 de abril, 2004. Aceptado en versión corregida el 22 de septiembre, 2004.

${ }^{1}$ Comité de Insuficiencia Renal Aguda de la Sociedad Chilena de Nefrología. ${ }^{2}$ Unidades de Nefrología y de ${ }^{3}$ Epidemiología, Clínica Dávila. ${ }^{4}$ Unidad de Diálisis, Clínica Las Condes.

anternos de Medicina, Facultad de Medicina, Universidad de los Andes. Santiago de Chile.

Correspondencia a: Dr. Antonio Vukusich C. Clínica Dávila, Recoleta 464, Santiago. Teléfono: 7308021. Fax: 7308885.

E mail: dirmedica@davila.cl 
$\mathrm{D}$ esde los ataques a Londres durante la segunda guerra mundial ${ }^{1}$, los estudios epidemiológicos internacionales sobre insuficiencia renal aguda (IRA) han aportado información acerca de las causas, características, incidencia y prevalencia de IRA en determinados grupos de pacientes ${ }^{2-5}$; y también acerca de los resultados de las terapias de reemplazo renal (TRR) utilizadas; ya sea peritoneodiálisis, hemodiálisis y técnicas continuas de reemplazo renal (TCRR) $)^{6,7}$. El estudio reciente más completo, corresponde a un estudio español, que siguió prospectivamente durante 9 meses todos los casos de IRA que ingresaron a los hospitales de la comunidad de Madrid $^{8}$.

Por otra parte, la revisión de la literatura nacional muestra que la mayoría de los trabajos realizados en nuestro país corresponden a estudios descriptivos, de casos hospitalarios, en una época en que el aborto séptico era frecuente y la diálisis no estaba bien desarrollada ${ }^{9-12}$. Otros, comunican casos inusuales de IRA, como nefritis intersticial aguda ${ }^{13,14}$, necrosis cortical renal ${ }^{15}$, loxocelismo cutáneo-visceral ${ }^{16,17}$ o IRA en grupos especiales, como pacientes de mayor edad ${ }^{18}$. También ha sido comunicada IRA asociada a inmunoterapia para el tratamiento del cáncer ${ }^{19}$. Un estudio prospectivo publicado en 1996 aporta información valiosa sobre las características y las diferencias en la evolución y el pronóstico de la IRA entre pacientes sépticos y no sépticos en una Unidad de Cuidados Intensivos de la V región ${ }^{20}$. Sin embargo, no se dispone de datos actualizados que permitan conocer la incidencia, causas, variedades clínicas o pronóstico de la IRA que pudieran ser útiles para desarrollar estrategias preventivas o para mejor disponer los recursos para su tratamiento en nuestro país.

Realizar estudios epidemiológicos extensos y complejos en nuestro medio, tiene importantes restricciones por las dificultades para obtener información oportuna, completa y confiable. Por otra parte, los pacientes con IRA de mayor impacto en el sistema sanitario -debido a su mal pronóstico y alto consumo de recursos- son aquellos que requieren de TRR. Por lo tanto, decidimos estudiar la epidemiología de la IRA sólo en este grupo de pacientes. En este trabajo se presentan los resultados de un estudio multicéntrico, prospectivo de IRA grave definida como aquella que requirió de TRR y cuyo propósito fue hacer un aporte al conocimiento de la epidemiología actual de esta enfermedad en nuestro país.

\section{PACIENTES Y MÉTOdO}

Diseño. Durante un período de seis meses (junio a noviembre de 2001), se estudiaron en forma prospectiva todos los casos de IRA que requirieron TRR ocurridos en pacientes adultos ingresados en 10 hospitales de la Región Metropolitana (RM). Cuatro de ellos eran hospitales públicos, cuatro privados y dos universitarios, con un total de 4.094 camas. Todos los grandes hospitales de la RM que cuentan con TRR para tratar la IRA fueron invitados a participar del estudio, no pudiendo incorporarse aquellos que, por diferentes razones, no estaban en condiciones de aportar información segura. Los pacientes menores de 18 años y aquellos con insuficiencia renal crónica con creatininemias basales $>2,5 \mathrm{mg} / \mathrm{dl}$ fueron excluidos del estudio.

En cada uno de los hospitales participantes, un nefrólogo coordinador entrenado y al tanto de los objetivos del estudio, fue el encargado de registrar, en hojas individuales de protocolo, los datos de todos los casos de IRA que necesitaron TRR, ocurridos en cualquier Servicio de su hospital, durante el período de estudio y de enviar la información a los investigadores para su análisis. Para estandarizar la información se utilizó un cuestionario que incluyó una serie de variables tanto cuantitativas como cualitativas, el cual era fácil de responder. Las variables incluidas en el cuestionario fueron: sexo, edad, lugar de origen (comunitaria u hospitalaria), causas de IRA, formas de presentación (oligúrica: $<400 \mathrm{ml} /$ día por más de 48 h vs no oligúrica), TRR utilizada, función renal al egreso y mortalidad. La hemodiálisis intermitente (HDI) fue definida como aquella que se realizaba 3 ó 4 veces a la semana por un período de tiempo menor o igual a 4 h por sesión. Las TCRR consistieron en hemofiltración o hemodiafiltración convencional continua. La hemodiálisis diaria prolongada (HDP) fue aquella que se practicó 50 más días a la semana por más de 4 horas. El número de camas y de egresos hospitalarios de cada hospital correspondiente al período estudiado se obtuvo de los datos oficiales del Departamento de Estadísticas Sanitarias del Minis- 
terio de Salud. Las causas de IRA grave se ordenaron en los siguientes 13 grupos, de acuerdo con un protocolo desarrollado por el Comité de IRA de la Sociedad Latinoamericana de Nefrología e Hipertensión: 1. Sepsis, definida según Conferencia Consenso ${ }^{21} ; 2$. Cirugía; 3. Infectocontagiosas (leptospirosis, hantavirus, malaria, cólera); 4. Isquemia renal (trauma, hipovolemia, shock no séptico); 5. Toxicidad exógena (medios de contraste radiológico, antibióticos, antiinflamatorios no esteroidales (AINE), bloqueadores de la enzima de conversión de la angiotensina, antagonistas de los receptores de angiotensina, paraquat, hongos, etc.); 6. Toxicidad endógena (pigmentos, cristales, lisis tumoral, etc.); 7. Glomerulonefritis/ Vasculitis; 8. Loxocelismo; 9. Nefritis intersticial alérgica; 10. Enfermedad hipertensiva del embarazo; 11. Síndrome hemolítico urémico (SHU); 12. Obstrucción vascular renal; y, 13. Obstrucción de las vías urinarias.

Estadística. Los test de t de Student y Chi cuadrado fueron usados respectivamente para comparar las variables cuantitativas y cualitativas.

\section{RESULTADOs}

Incidencia y formas de presentación. Durante los seis meses del estudio se presentaron un total de 114 casos de IRA grave en los 10 hospitales participantes, de los cuales 65 (57\%) correspondieron a pacientes de sexo masculino y 49 (43\%) al sexo femenino. La edad media fue de 61,2 años, con un rango entre 18 y 87 años. El 38\% de los pacientes tenía más de 69 años de edad. El número total de egresos de los hospitales estudiados durante el período fue de 110.399; por lo tanto, la incidencia de IRA grave fue de 1,03 casos por 1.000 egresos con un rango de 0,3 a 1,4 por 1.000, dependiendo de la complejidad de los diferentes centros hospitalarios. El número de egresos señalado corresponde, a su vez, a $54 \%$ del total de los egresos de todos los hospitales de la Región Metropolitana que estaban en condiciones de realizar alguna forma de TRR. En 79 pacientes $(69,3 \%)$ la IRA grave apareció durante la estancia en el hospital, mientras que los 35 casos restantes $(30,7 \%)$ se iniciaron en la comunidad. Sesenta y cuatro casos se presentaron de forma oligúrica $(56,1 \%)$ y los otros 50 de forma no oligúrica $(43,9 \%)$.

Etiologías. En la mayoría de los pacientes: 69 casos $(60,5 \%)$, se asociaron dos o más causas de IRA grave, mientras que en 45 (39,5\%), sólo se informó de una causa. Sepsis resultó ser la causa más frecuente estando presente, ya sea como causa única o asociada con otras, en 58 (50,9\%) de los 114 casos. La siguieron, en forma aislada 0 combinada con otras causas, isquemia en 53 $(46,5 \%)$, cirugía en $30(26,3 \%)$, toxicidad exógena en 28 casos (24,5\%), en 26 de los cuales se hallaba asociada a otras causas, y toxicidad endógena en 13 casos (11,4\%). (Figura 1). La isquemia fue la causa única en 8 pacientes. De los 30 casos con

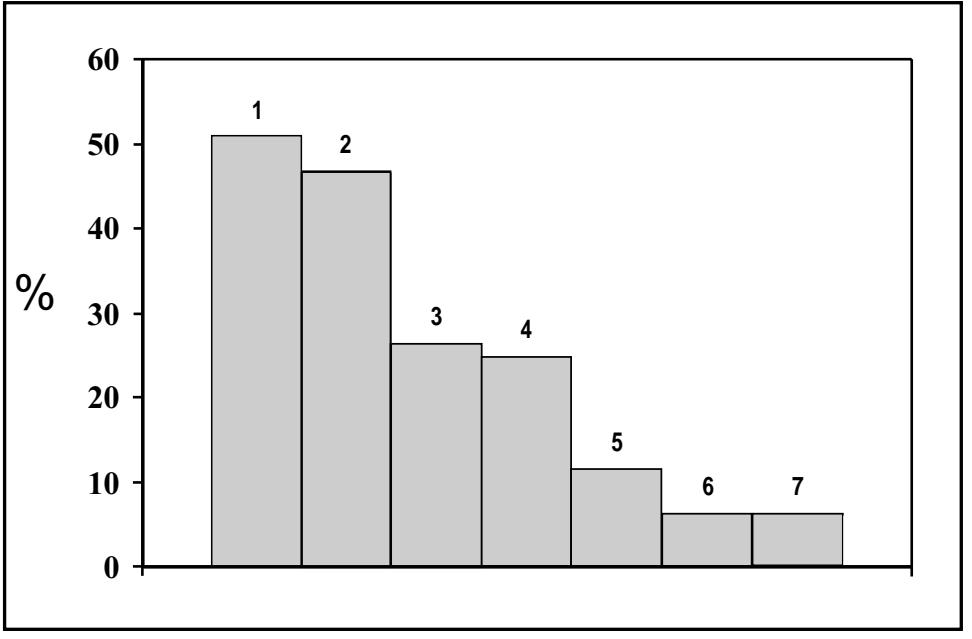

Figura 1. Causas de IRA grave en 114 pacientes de 10 hospitales de la Región Metropolitana.

Leyenda: (1) Sepsis; (2) Isquemia; (3) Cirugía; (4) Toxicidad exógena; (5) Toxicidad endógena; (6) Glomerulonefritis/Vasculitis; (7) Uropatía obstructiva. En 69 pacientes $(60,5 \%)$ se presentaron combinadas dos o más causas. 
IRA postoperatoria, 14 (47\%) habían sido sometidos a cirugía cardíaca. Las causas más frecuentes de toxicidad exógena fueron los antibióticos nefrotóxicos con 11 casos (39,3\%); seguidos de los AINE y medios de contraste radiológico con 4 casos cada uno (14,3\%). Catorce de los 28 pacientes con toxicidad exógena (50\%), presentaron simultáneamente isquemia como factor de riesgo. La toxicidad exógena se presentó como causa única sólo en dos pacientes: el primero con una severa tubulopatía secundaria al uso masivo de manitol en el postoperatorio de un tumor cerebral, y el segundo, por el uso de AINE. En 10 de los 13 casos de toxicidad endógena, ésta aparece combinada con otros factores de riesgo (77\%). Las causas más frecuentes de toxicidad endógena fueron la nefropatía por pigmentos (mioglobina, bilirrubina) en 5 casos $(38,5 \%)$ y el síndrome de lisis tumoral en $4(30,7 \%)$. El daño glomerular agudo y la uropatía obstructiva representan, cada uno, 7 casos, con 6,1\% respectivamente. En 10 de los 114 pacientes estudiados, se presentaron enfermedades que no estando asociadas a otros factores de riesgo suelen determinar IRA por mecanismos diferentes al daño tubular agudo: 2 casos de enfermedades infectocontagiosas (1 Hantavirus y 1 Malaria); 2 casos de uropatía obstructiva, y 6 casos de glomerulonefritis/vasculitis; de manera que podemos inferir (sin contar con la confirmación histológica) que la necrosis tubular aguda (NTA) tóxica isquémica o ambos factores se presentó en 104 de los 114 pacientes lo que corresponde al 91,2\%. Esto representa una incidencia de NTA de 0,94 casos por 1.000 egresos.

Durante el período de estudio no se encontraron casos de IRA grave secundaria a SHU, loxocelismo, enfermedad hipertensiva del embarazo, nefritis intersticial alérgica, u obstrucción vascular renal en ninguno de los 10 hospitales estudiados.

Terapias de reemplazo renal (TRR). La HDI fue el tratamiento más utilizado, siendo tratados de esta forma 75 pacientes (65,8\%). En 33 casos (29\%) se aplicaron TCRR, mientras que la HDP se utilizó sólo en 2 pacientes (1,7\%) (Figura 2). En 4 casos (3,5\%) se combinó HDI con TCRR.

Mortalidad. La mortalidad global fue de 44,7\% (51 pacientes), siendo mayor en los casos oligúri$\cos (54 \%$ vs $33 \% ; \mathrm{p}=0,048)$ y no mostró variación con el sexo, la edad, el origen o las causas de IRA ni la TRR utilizada. Todos los pacientes que fallecieron lo hicieron con IRA presente. De los 63 pacientes que sobrevivieron, seis presentaban algún grado de disfunción renal al egreso del hospital $(9,5 \%)$.
Figura 2. Tipos y porcentajes de uso de terapias de reemplazo renal en 114 pacientes con IRA grave.

Leyenda: HDI: hemodiálisis intermitente; TCRR: técnicas continuas de reemplazo renal; HDP: hemodiálisis diaria prolongada.

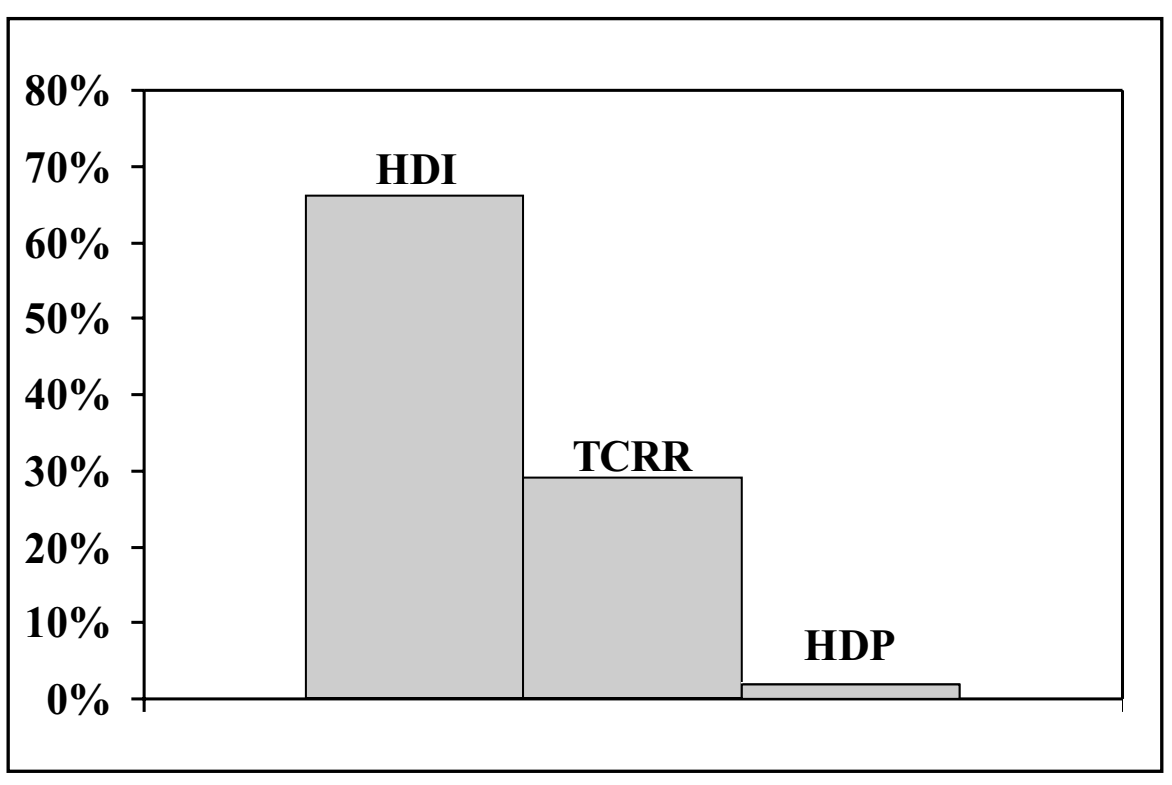




\section{DisCUSIÓN}

En este estudio prospectivo se comunica la incidencia, formas de presentación, causas, tratamiento y mortalidad de la IRA grave en una población adulta de diez hospitales de la RM durante un período de seis meses. Por las características del diseño del mismo, se trata de un análisis descriptivo.

La IRA grave, es decir, aquella tratada con alguna forma de TRR tuvo una incidencia de 1,03 casos por 1.000 egresos, cifra mayor a la que se desprende del gran estudio comunitario de Liaño ${ }^{8}$. Este encontró que el 1,5 por 1.000 de los pacientes admitidos a los hospitales terciarios de la comunidad de Madrid desarrollaban alguna forma de IRA, pero de éstos, sólo 36\% requería TRR. Por otra parte, estudios más pequeños, utilizando como punto de partida sólo pacientes hospitalizados han mostrado que, en ellos, la incidencia de IRA oscila alrededor de 3\%2,3. Es plausible pensar que -en un caso y otro- las diferencias en las cifras reflejen, por una parte, las diferencias en las poblaciones estudiadas y, por otra, las variaciones en la metodología empleada. En nuestro caso el criterio de inclusión de los pacientes no fue el lugar de origen de la IRA -comunitario u hospitalario- sino su gravedad, es decir la necesidad del uso de TRR. Sin embargo, la mayoría de los pacientes se complicó de IRA durante la hospitalización, destacando la convergencia de dos o más causas desencadenantes. La sepsis, la cirugía, especialmente intervenciones cardíacas, la isquemia renal y la administración de nefrotóxicos, como antibióticos, AINE y medios de contraste radiológico, emergen como las causas más importantes, muchas de ellas prevenibles. Cabe destacar la posibilidad de disminuir la incidencia de IRA mediante la prevención de la isquemia renal y la elección de antibióticos y analgésicos no tóxicos. También es posible disminuir la incidencia de nefropatía inducida por medios de contraste gracias a una adecuada expansión del volumen extracelular22 ${ }^{22}$ La administración de acetil-cisteína también se ha reportado útil en estas condiciones $^{23}$. La IRA, como lo demuestra este estudio, es la consecuencia, en gran medida, del uso de técnicas modernas de diagnóstico y tratamiento que requieren de estrategias preventivas para disminuir su impacto. Sin duda, el aborto séptico ha sido desplazado como causa fundamental de IRA, sin embargo, la sepsis, de diversos orígenes, continúa siendo la primera condición que requiere de TRR en nuestro país. La alta incidencia de NTA es consistente con estudios intemacionales ${ }^{2-5,8}$ pero debe ser tomada con cautela debido a que se trata de un estudio clínico donde este diagnóstico se efectuó en forma presuntiva.

En todo el mundo la mortalidad en la IRA grave es aún muy alta, especialmente en los pacientes críticos. La mortalidad global de 44,7\% descrita en este estudio, está en el rango inferior de lo comunicado pero incluye a toda la población hospitalaria y no sólo a los pacientes de Unidades de Pacientes Críticos en donde la literatura señala mortalidades que superan el 70\%. Este estudio no incluyó pacientes con IRA que necesitando de TRR no la recibieron (enfermos terminales o extremadamente graves), porque un criterio de inclusión era haber recibido TRR. En el estudio epidemiológico efectuado en Madrid se reporta una mortalidad de $65,9 \%$ en pacientes con IRA tratada con diálisis ${ }^{8}$. Encontramos una diferencia de mortalidad significativamente mayor al comparar a los pacientes oligúricos con los no oligúricos lo que también está de acuerdo con los datos de la literatura, y permite inferir que las medidas de protección renal y el adecuado manejo de los factores determinantes del flujo sanguíneo renal se asocian a un menor daño tubular y permiten una mejor sobrevida.

El tipo de TRR utilizada no influyó en el pronóstico de nuestra población -como ha ocurrido también con otras comunicaciones- pero en este estudio, se analizan en conjunto pacientes críticos con falla multiorgánica y pacientes no críticos como aquellos portadores de glomerulopatías o uropatías obstructivas. La dosis de tratamiento dialítico -más que la técnica utilizada para lograrla- parece influir directamente en el pronóstico vital de enfermos críticos, sin embargo, la información es aún insuficiente. Un estudio de Schiffl -ampliamente citado pero cuyos datos han sido cuestionados- describe una reducción de la mortalidad de $46 \%$ a $28 \%$ al comparar en forma prospectiva hemodiálisis en días alternos de 3,4 horas de duración promedio, con diálisis diaria de 3,3 horas de duración promedio 24 . En nuestro estudio no se midió la dosis de TRR recibida por los pacientes, sin embargo, se puede inferir un significativo esfuerzo para lograr una mayor dosis de diálisis mediante TCRR, las que han tomado un 
lugar cada vez más frecuente, y con HDP, que aunque practicada en sólo dos casos es la técnica disponible en nuestro país que permite prescribir las mayores dosis de diálisis que hoy pueden ser administradas. Un hallazgo importante es el número de pacientes con algún grado de disfunción renal al alta. El protocolo de estudio no incluyó mediciones del tiempo de duración de la IRA, por lo tanto, no podemos relacionarlo con la TRR utilizada, sin embargo, la mortalidad no varió con las distintas formas de TRR. Es posible que, en algunos casos de NTA, la recuperación completa de la función renal después de una IRA grave tome más tiempo de lo previsto y que, en otros, se mantenga un grado de falla renal irreversible, lo que haría de los pacientes, que han sobrevivido a una IRA que requirieron TRR, una población en riesgo de desarrollar insuficiencia renal crónica.

En resumen, este estudio demuestra una incidencia de IRA grave en la RM de 1,03 casos por 1.000 egresos. Es una condición patológica adquirida fundamentalmente en el hospital y originada por la confluencia de varios factores entre los que destacan la sepsis, las intervenciones quirúrgicas, especialmente la cirugía cardíaca, la isquemia y la administración de nefrotóxicos. El mejor pronóstico se presenta en pacientes con la forma no oligúrica y la mortalidad afecta a aproximadamente la mitad de los enfermos. El tipo de TRR utilizado en este grupo de pacientes no influyó en la mortalidad observada.

\section{REFERENCIAS}

1. Bywaters E. 50 years: The crush syndrome. BMJ 1990; 301: 1412-5.

2. Hou S, Bushinsky D, Wish J, Cohen J, Harrington J. Hospital acquired renal insufficiency: A prospective study. Am J Med 1983; 74: 243-8.

3. Shusterman N, Strom B, Murray T, Morrison G, WeST S, MAISLN G. Risk factors and outcome of hospital acquired acute renal failure. Am J Med 1987; 83: 65-71.

4. FeEst T, Round A, Hamad S. Incidence of severe acute renal failure in adults. Results of a community based study. BMJ 1993; 306: 481-3.

5. Kaufman J, Dhakal M, Patel B, Hamburger $R$. Community acquired acute renal failure. Am J Kidney Dis 1991; 17: 191-8.

6. JAKOB S, Frey F, WeHLinger D. Does continuous renal replacement therapy favourably influence the outcome of the patients? Nephrol Dial Transplant 1996; 11: 1250-5.

7. Hakim $\mathrm{R}$, Wingard $\mathrm{R}$, Parker $\mathrm{R}$. Effect of the dialysis membrane in the treatment of patients with acute renal failure. N Engl J Med 1994; 331: 1338-42.

8. Liaño F, Pascual J. Madrid Acute Renal Failure Study Group: Epidemiology of acute renal failure: A prospective, multicenter, community based study. Kidney Int 1996; 50: 811-8.

9. Kuzmanic A, Alvo M, Faivovich A, Melfi M, GonzÁLEZ F, SABA R. Insuficiencia Renal Aguda. Rev Méd Chile 1965; 93: 302-8.

10. Katz E. Diagnóstico y tratamiento de la insuficiencia renal aguda. Rev Méd Chile 1973; 101: 966-71.

11. Kuzmanic A, Alvo M, Faivovich A, Philppi V. Insuficiencia renal aguda: Experiencia en 115 pacientes. Rev Méd Chile 1976; 104: 713-7.

12. Alvo M, Kuzmanic A, Faivovich A, Plubins L, Meifi M, GoNZÁLEZ F ET AL. Insuficiencia renal aguda: Experiencia de 22 años. Rev Méd Chile 1981; 109: 420-7.

13. Flores JC, Gehrung E, Rosenberg H. Insuficiencia renal aguda asociada a nefritis intersticial aguda. Rev Méd Chile 1982; 110: 37-42.

14. Mônckeberg G, Vukusich A, Valls G, Rosenberg H. Nefritis intersticial aguda y lamotrigina. Rev Méd Chile 2004; 132: 742-6.

15. FuENZALDA E. Insuficiencia renal aguda por necrosis cortical renal. Dos casos clínicos de pacientes sobrevivientes. Rev Méd Chile 1991; 119: 793-7.

16. VuKusich A. Loxocelismo cutáneo-visceral mortal. Bol Chile Parasitol 1962; 17: 25-8.

17. González C, SafFie A, Fardelia C, Contreras A. Insuficiencia renal aguda en loxocelismo cutáneo-visceral: 11 casos. Rev Méd Chile 1986; 114: 1155-9.

18. Jalil R, Downey P, Jara A, Castellón JM, Hoyl T, MeLia JM, Vial S. Insuficiencia renal aguda en 
pacientes mayores: evaluación de factores pronósticos. Nefrología 1995; XV-4: 343-8.

19. Morales J, Vukusich A, Gutiérrez J. Insuficiencia renal aguda asociada a inmunoterapia por cáncer metastático. Rev Méd Chile 1994; 122: 1085-6.

20. Vega J, Borja H, Videla C, Aldunate T, Clavero R, ECHEVERRía $C$ ET AL. Insuficiencia renal aguda en pacientes con y sin sepsis: pronóstico y evolución. Rev Méd Chile 1996; 124: 938-46.

21. Bone R, Balk R, Cerra F, Dewnger P, Fein A, Knaus W ET AL. ACCP/SCCM Consensus Conference. Definition for sepsis and organ failure and guide- lines for the use of innovative therapies in sepsis. Chest 1992; 101: 1644-55.

22. Solomon R, Werner C, Mann D, D'elua J, Silva P. Effects of saline, mannitol and furosemide on acute changes in renal function induced by radiocontrast agents. N Engl J Med 1994; 331: 1416-20.

23. Tepel M, Van Der Giet M, Schwarzfeid C, Laufer U, LERMANN D, ZIDEK W. Prevention of radiographic contrast agent induced reductions in renal function by acetylcysteine. N Engl J Med 2000; 343: 180-4.

24. SCHIFFL H, LANg SM, Fischer R. Daily hemodialysis and the outcome of acute renal failure. N Engl J Med 2002; 346: 305-10.

\section{Agradecimientos}

Los autores agradecen a los siguientes nefrólogos y hospitales participantes en este estudio: Dr. Erico Segovia, Hospital JJ Aguirre; Dra. Mireya Ortiz, Hospital UC; Dr. Gonzalo Valls, Clínica Dávila; Dr. Carlos Zehnder, Clínica Las Condes; Dr. Alberto Fierro, Clínica INDISA; Dr. Ronald Weinstein, Hospital Militar; Dr. Mauricio Espinoza, Clínica Alemana y Hospital del Salvador; Dr. David Benavente, Hospital Barros Luco-Trudeau y Dr. Enrique Quintana, Hospital Padre Hurtado. 